\title{
Bulk Properties of Syndiotactic 1,2-Polybutadiene. II. Mechanical Properties of Uniaxially and Biaxially Stretched Films in Relation to Molecular Orientation
}

\author{
Yutaka OвAтA, Chikara Homma, and Chikao Tosaki \\ Research Laboratory, Japan Synthetic Rubber Co., \\ 7569, Ikuta, Tama-ku, Kawasaki, Japan. \\ Nobuhide SHIRAISHI \\ Yokkaichi Research Laboratory, Japan Synthetic Rubber Co., \\ 100, Kawajiri-cho, Yokkaichi, Mie, Japan.
}

(Received July 5, 1974)

\begin{abstract}
This paper discusses the mechanical properties of syndiotactic 1,2-polybutadiene with $25-\%$ crystallinity in relation to the unrecoverable strain, stress relaxation, and orientation properties under large deformation in the range of melting temperature from 30 to $90^{\circ} \mathrm{C}$.

The following results were obtained:

(1) The mechanical properties of the stretched films obtained by large uniaxial and biaxial deformations depend mainly on two factors: A) the ambient temperature during the stretching, and B) the types of deformation.

(2) This dependence may be ascribed to the molecular orientation of the amorphous chain and some type of disintegration of the crystal structure; the orientation of crystal axes seems to be contribute little for such a polymer with a low degree of crystallinity. KEY WORDS 1,2-Polybutadiene / Orientation Coefficient / Film / Stretching / Biaxial Strain /
\end{abstract}

When a film sample of 1,2-polybutadiene is stretched beyond the yielding point, it does not return to the original state after the removal of the external force applied. The mechanical properties also become different from those of the initial (unstretched) specimen.

The main purpose of the present study is to obtain some information which may be useful in designing a process of making stretched film with desirable mechanical properties. On the analogy of a similar problem in melt spinning, the main factors affecting the mechanical properties of the stretched film may be (1) temperature during stretching, (2) degree and type of deformation, (3) strain rate, and (4) cooling conditions after the stretching. Very few studies, however, have been done to connect the final properties with the conditions of making the stretched film, especially in the case of semi- crystalline polymers.

Another interest is in obtaining the relationship between the mechanical properties and the change of the morphology. It is well known that the orientation of molecular chains affects the stiffness and strength of the stretched film or fiber., ${ }^{3,8}$

In this study, stress and strain under the uniaxial and different types of biaxial strain histories were observed in the temperature range of crystal fusion by using an Instron type tensile testing machine and a biaxial tensile testing machine called Bistron. ${ }^{4}$ Further, stress relaxation behavior after the release of the stretching and the unrecoverable strain after the release of the stress were also observed. The orientation of molecular chain axes in both crystalline and amorphous phases was observed by $\mathrm{X}$-ray diffraction and angular distribution of polarized 
components of fluorescent intensity, respectively. The results are discussed together in connection with the final properties of the stretched film.

\section{PREPARATION OF SAMPLES}

A sample of 1,2-polybutadiene with $25-\%$ crystallinity (designated by $\mathrm{S} 25$ in a previous paper $^{1}$ ) was used in this study. Some sheets of film were prepared by two different methods; press molding, mentioned in the previous paper, and extrusion with a T-die. The thickness of the film prepared by press molding was about $0.8 \mathrm{~mm}$, the one by extrusion was about $0.2 \mathrm{~mm}$. The press molded specimen (designated hereafter by Pms) was cut into a long rectangular or ring shape and used for the uniaxial experiment. Specimens made with the T-die (designated hereafter by Tdf) were cut into a square of $12.4 \times$ $12.4 \mathrm{~cm}^{2}$ and marked by a stamp with two sets of nine parallel lines, so as to form a square grid of $1.5-\mathrm{cm}$ spacing; they were used for the biaxial stretching. In the results of X-ray diffraction analysis and the angular distribution of polarized components of fluorescene intensity, no anisotropy was observed in either specimen. Further, the degrees of crystallinity of Tdf and Pms were found by X-ray diffraction analysis to be the same within experimental error. The Tdf, however, was found to be slightly anisotropic in the stress and strain relation. Figure 1

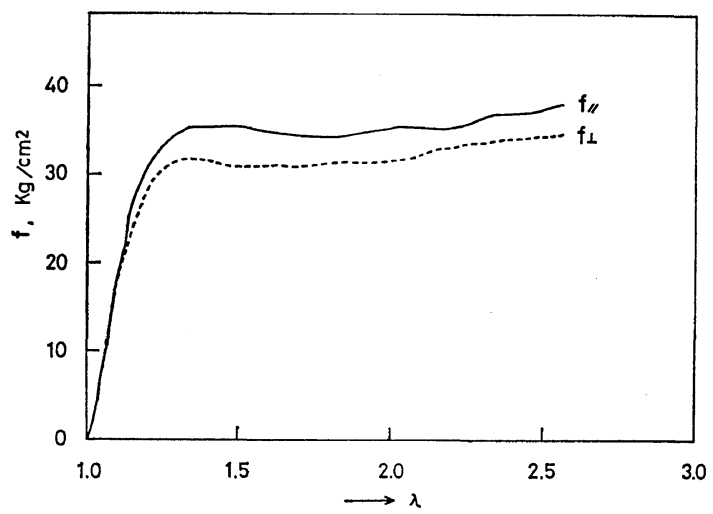

Figure 1. Stress-strain curves of T-die film parallel to machine direction (-) and perpendicular to machine direction $(---)$, measured at $40^{\circ} \mathrm{C}$ at constant rate of strain $\dot{\gamma}=12.5 / \mathrm{min}$.

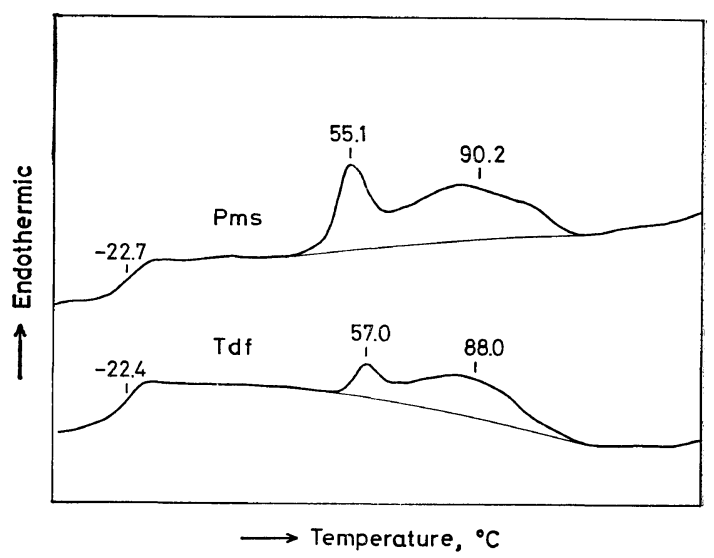

Figure 2. DSC thermograms of $\mathrm{T}$-die and press molded film scanned at $16^{\circ} \mathrm{C} / \mathrm{min}$.

shows stress-strain curves of two test pieces of Tdf under the uniaxial elongation: one was cut in the direction parallel to the machine direction and the other was cut perpendicular to the machine direction. Comparing the two stress-strain curves in the figure, a slight difference was found in the flat region of the stresses. It will be shown later that anisotropy as slight as this is negligible when it is compared with the change of the mechanical properties induced by the deformations in this study. No considerable difference was seen in the stressstrain curves of Pms.

Figure 2 shows DSC thermograms of Tdf and Pms at a heating rate of $16^{\circ} \mathrm{C} / \mathrm{min}$. They indicate that both specimens have nearly the same thermal histories.

\section{DESIGN OF STRAIN HISTORY}

It is expected that the mechanical properties of stretched film are closely related to the following properties in the process of making the film: stress-strain, stress relaxation in the process of stretching, and unrecoverable strain after the stretching. In order to investigate some of the mechanical behavior in the process of the stretching, the following experimental design was made; a schematic representation is shown in Figure 3. In the figure, a test specimen is set into the testing apparatus and annealed for $10 \mathrm{~min}$ (stage I), stretched up to the predetermined amount of strain at a constant rate 
Bulk Properties of Syndiotactic 1,2-Polybutadiene. II.

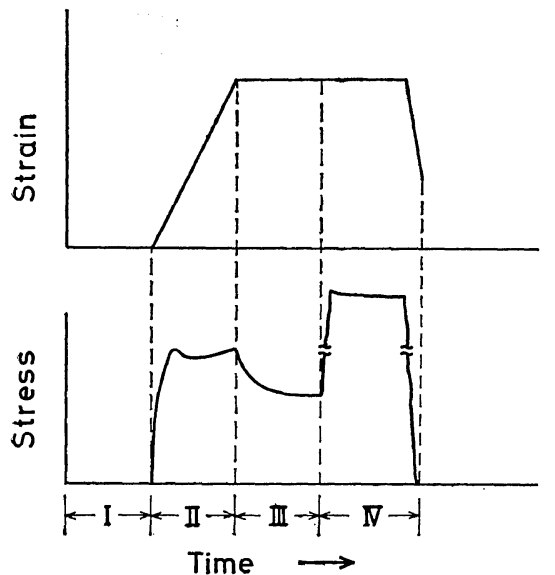

Figure 3. Schematic representation of controlling strain and stress designed for the investigation of the mechanical properties of a specimen under large deformation.

(stage II), kept at the strain state for $5 \mathrm{~min}$ to observe the stress relaxation (stage III), quenched by the vapor of liquid nitrogen or cooled slowly by room air, while the strain is kept to the previous state (the stress increases rapidly with decreasing temperature), and then allowed to shrink by releasing the external stress (stage IV).

In stages II and III, the stress was measured as a function of the time. In stage IV, stretch ratios in two orthogonal machine directions $(X$ and $Y$ ) were measured after cooling was completed and the specimens were exposed to room air; then the specimens were released from force for $10 \mathrm{~min}$ and the stretch ratios were measured again. The former was designated by $\lambda_{\mathrm{m}}$ and the latter by $\lambda_{\mathrm{r}}$.

In the biaxial stretching, various ways of stretching are possible by controlling strains of both $X$ and $Y$ directions independently. In this study, biaxial stretching in one step and biaxial stretching it two steps were performed. Changes in stresses $f_{x}$ and $f_{y}$ under the biaxial stretchings in one step and in two steps are shown in Figure 6 with changes of $\lambda_{x}$ and $\lambda_{y}$.

In the measurements of X-ray diffraction and fluorescene measurements, the stretched specimen was clamped by a pair of ring-shaped chucks after it was quenched (in stage IV). Stretch ratios $\lambda_{\mathrm{m}}$ and $\lambda_{\mathrm{r}}$ were obtained by measuring the distances between two marks before and after deformations. The details of each experiment will be described in the respective sections.

\section{STRESS AND STRAIN UNDER LARGE DEFORMATION}

The Dynamic tensile Young's modulus $E^{\prime}$ and $\tan \delta$ responsible for the viscoelasticity under an infinitesimal deformation were shown in a previous paper. ${ }^{1}$ From this data, it was expected that the stress-strain behavior under large deformation would depend considerably on the temperature. And such temperature dependence of the stress-strain curve was observed under the uniaxial deformation using ring-shaped specimens of Pms. Figure 4 shows hysteresis loops under a constant rate of strain, $1.25 / \mathrm{min}$, at temperatures ranging from 30.7 to $79.2^{\circ} \mathrm{C}$. The decrease of the stress with increase of the temperature may be easily understood as the decrease of the degree of crystallinity with the temperature, as has been examined in the previous paper. Further, it was noticed that the unrecoverable strain observed in each loop becomes smaller with increasing temperature. This will be discussed later with the data from other types of deformation.

The dependence of stress-stain curves on the rate of strain was observed at temperatures ranging from 29 to $83^{\circ} \mathrm{C}$ and was found to be very small. For example, the values of the $100-\%$

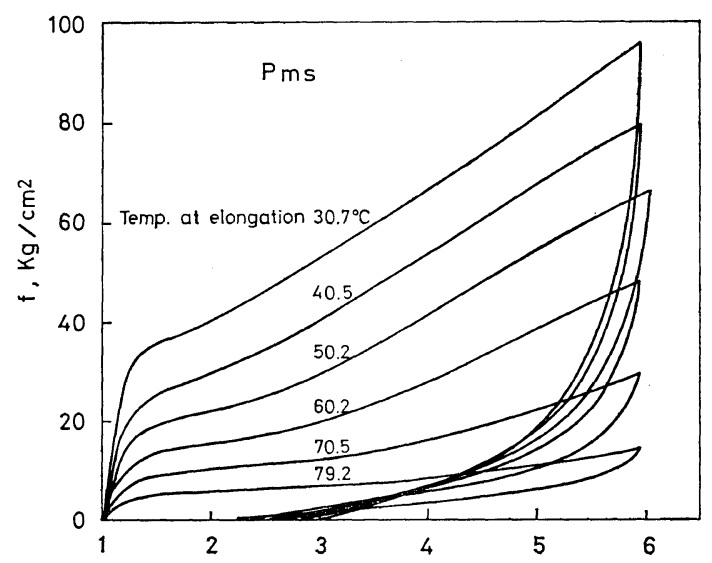

Figure 4. Temperature dependence of hysteresis loop of Pms measured at temperatures ranging from 30.7 to $79.2^{\circ} \mathrm{C}$ at constant rate of strain $\dot{\gamma}=12.5 / \mathrm{min}$. 


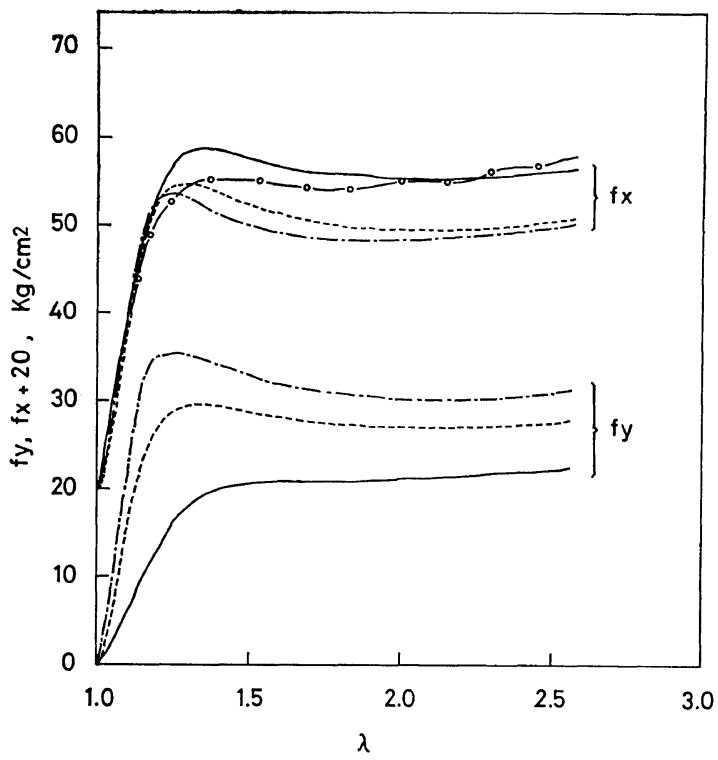

Figure 5. Stresses $f_{x}$ and $f_{y}$ under uniaxial and various types of biaxial deformations: $f_{x}$ (ps.), (一); $f_{x}$ (uni.), (-○-); $f_{x}$ (nb.), (---); $f_{x}$ (ub.), (--). The same identifications are applied to $f_{y}$.

modulus at the strain rates $\dot{\gamma}=12.5 / \mathrm{min}$ and $2.5 / \mathrm{min}$ were 28.1 and $27.0 \mathrm{~kg} / \mathrm{cm}^{2}$, at $41^{\circ} \mathrm{C}$.

Another factor affecting the stress-strain relation may be the type of the deformation. Figure 5 shows engineering stresses $f_{x}$ and $f_{y}$ under uniaxial and various types of biaxial deformations measured at $40^{\circ} \mathrm{C}, \dot{\gamma}=1.25 / \mathrm{min}$. Designations of the stresses were made as follows:

$f$ (uni.): stress under uniaxial deformation.

$f_{x}$ (ps.): stress in $X$ direction under pure shear, i.e., $\lambda_{y}=1$.

$f_{x}$ (ub.): stress under equal biaxial, $i, e .$, $\lambda_{x}=\lambda_{y}$.

$f_{x}(\mathrm{nb}),. f_{y}$ (nb.): $\quad$ stresses $f_{x}$ and $f_{y}$ stretched in the orthogonal directions at different strains, such as $\lambda_{x}-1=k_{x} t, \quad \lambda_{y}-1=k_{y} t ; \quad k_{x}=$ $12.5 / \mathrm{min}, k_{y}=5.8 / \mathrm{min}$.

From the figure, the relation between $f_{x}$ and $\lambda_{x}$ is nearly independent of the type of deformation in the range of deformations less than $15 \%$. Beyond the yielding point, values of $f_{x}$ depend considerably on the types of deforma-

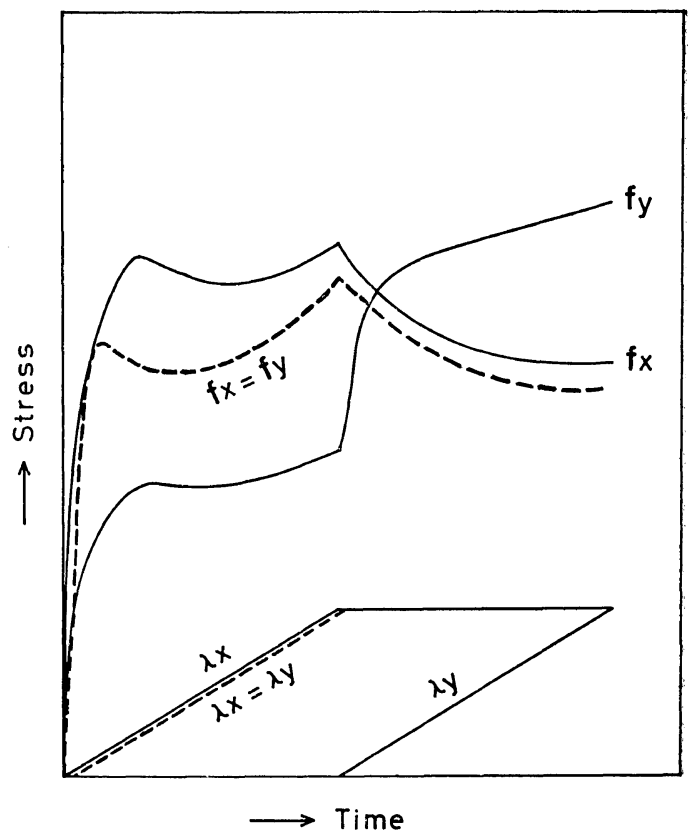

Figure 6. Stress-strain curves $f_{x}$ and $f_{y}$ of Tdf under biaxial extension in two steps ( - ) and in one step (-) and in one step (-- ) measured at $40^{\circ} \mathrm{C}$ at constant rate of strain $\dot{\gamma}=12.5 / \mathrm{min}$ and changes of $\lambda_{x}$ and $\lambda_{y}$ with time.

tion. The relations between the stresses were found to be

$$
f_{x} \text { (ps.) }>f_{x} \text { (nb.) }>f_{x} \text { (ub.) }, \quad f_{x} \text { (ps.) }>f_{x} \text { (uni.) } .
$$

Thus, the stress $f_{x}$ at pure shear is larger than those at uniform biaxial and uniaxial stretchings. In rubber vulcanizates, the following simple relation is valid for $f_{x}$ and $f_{y}$ under any type of deformation:

$$
f(a, b)>f(a, c) \text { for } b>c
$$

In order to illustrate how the stresses $f_{x}$ and $f_{y}$ change under equal biaxial deformations in one step and in two steps, typical curves of $f_{x}$ and $f_{y}$ are shown in Figure 6 as a function of time through the change of $\lambda_{x}$ and $\lambda_{y}$. In the deformation in one step, $\lambda_{x}$ is always equal to $\lambda_{y}$ and consequently $f_{x}=f_{y}$. In the deformation in two steps, $\lambda_{y}$ is fixed to unity until $\lambda_{x}$ attains to a fixed value and then $\lambda_{y}$ increases, while $\lambda_{x}$ being kept at the fixed value. The result of Figures 5 and 6 suggests that some kind of morphological changes of the specimen 
may arise in the course of stretching and that the degree of this change depends somewhat on the type of deformation. In order to investigate how the morphological change depends on the type of stretching and the stretching temperature, the following experiments were designed. Test specimens from Tdf were stretched biaxially in one step and in two steps at 30 , $35,40,50,60$, and $70^{\circ} \mathrm{C}$, according to the process shown in Figure 3. Test specimens of Pms with ring shape were stretched uniaxially at the corresponding temperatures in the same process as above. Stress relaxation behaviors were observed in the stress relaxation curves after the stretching along both $X$ and $Y$ directions were fixed. A ratio of the stress after 5 min stress relaxation $\left(f_{5}\right)$ to the stress at the beginning of the relaxation $\left(f_{0}\right)$ was measured for each stress time curve in the process III. The ratio $f_{5} / f_{0}$ defined is rather like a survival rate and it is unity for a completely elastic material.

A morphological change of the specimen induced by the stretching may be reflected by the unrecoverable strain after the removal of the external force. Thus the ratio $\lambda_{\mathrm{r}} / \lambda_{\mathrm{m}}$ was defined as a measure, where $\lambda_{\mathrm{m}}$, which is the maximum stretch ratio obtained, was set to be 6.0 and
2.5 for uniaxial and biaxial (for both $X$ and $Y$ directions) stretching, respectively. The reason for choosing these values of $\lambda_{\mathrm{m}}$ is not due to any concrete criterion but to the experimental demand that the specimen could be stretched without rupture over the range of temperature mentioned.

Figure 7 shows $\lambda_{\mathrm{r}} / \lambda_{\mathrm{m}}$ and $f_{5} / f_{0}$ of both the uniaxial and the biaxial stretching as a function of the ambient temperature at stretching. In the figure, it should be noted that the stress relaxation behavior $\left(f_{5} / f_{0}\right)$ does not depend on the type of deformation below $45^{\circ} \mathrm{C}$, but it does at higher temperatures. And it is easily seen that the unrecoverable strain $\lambda_{\mathrm{r}} / \lambda_{\mathrm{m}}$ of biaxial stretching is much larger than that of uniaxial stretching and that its dependence on the temperature at stretching is affected by the type of deformation. That is, the stress-strain behavior becomes more elastic with increase of the temperature in the case of uniaxial deformation, while it becomes more plastic in the case of biaxial stretching. This suggests that some kind of plastic flow or failure of initial structure may arise and will depend greatly on the stress field induced. Thus it is expected that stretched films with different mechanical properties can be produced by selecting the temperature and type of
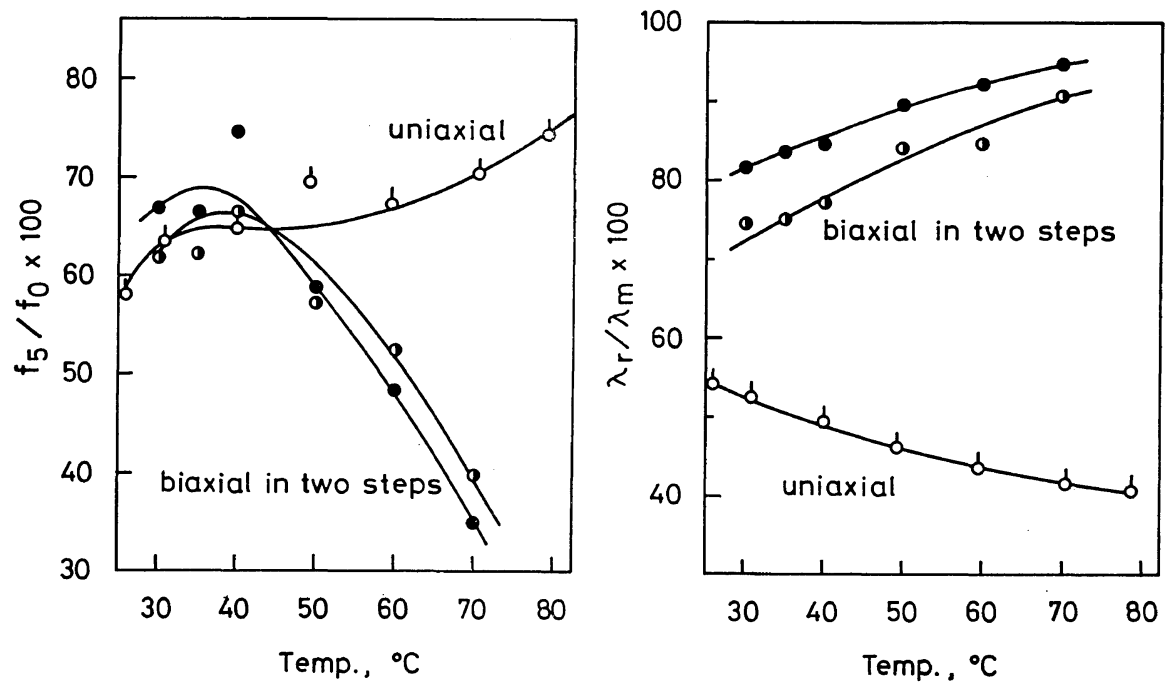

Figure 7. $\lambda_{\mathrm{r}} / \lambda_{\mathrm{m}}$ and $f_{5} / f_{0}$ in the process indicated by Figure 5 under uniaxial $(\bigcirc)$, biaxial in two steps cut along $Y$ direction (O) or along $X$ direction (O). Specimens in the figure were all quenched after the stress relaxation. 


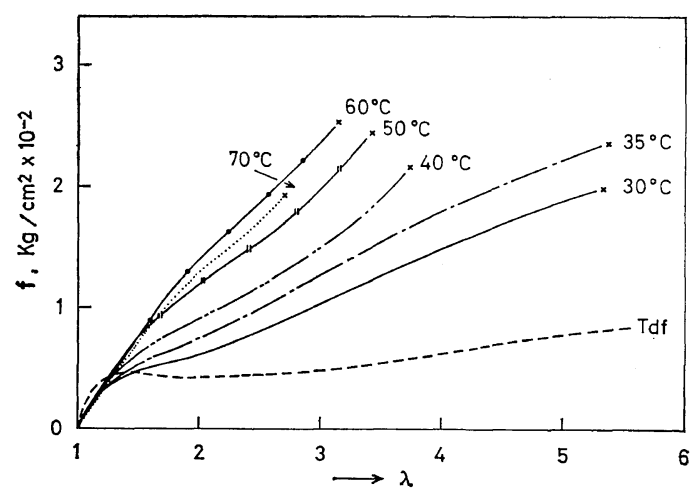

Figure 8. Stress-strain curves measured at room temperature of specimens cut from biaxially stretched film (biaxial in two steps, quenched) in the direction parallel to $Y$ axis.

deformation. Some mechanical properties discussed hitherto are those observed in the process of making stretched film.

The following discussions concern the mechanical properties of a stretched film which was obtained by the process shown in Figure 3. Test specimens were cut in a long rectangular shape in both $X$ and $Y$ directions from the stretched film. Stress-strain curves were observed at a constant rate of strain, $1.25 / \mathrm{min}$, at room temperature. Figure 8 shows a series of stress-strain curves of the test specimens cut in the $Y$ direction from the films stretched biaxially in two steps. Marks $x$ in the figure indicate failure points. The figure shows that stress-strain under the finite and ultimate deformation is greatly affected by the temperature at stretching. Some other factors affecting the mechanical properties, such as strain rate, cooling conditions, and type of strain history were observed; it was found that their contributions were relatively small compared to the effect of temperature at stretching. In order to illustrate the dependence of the stress-strain on the temperature at stretching and on the type of the deformation, 100-\% moduli were obtained by reading from the curves in Figure 8 and from the similar curves of specimens obtained by uniaxial stretching at various temperatures. They are plotted against the temperature at stretching in Figure 9.

Experimental results shown in Figures 7 and

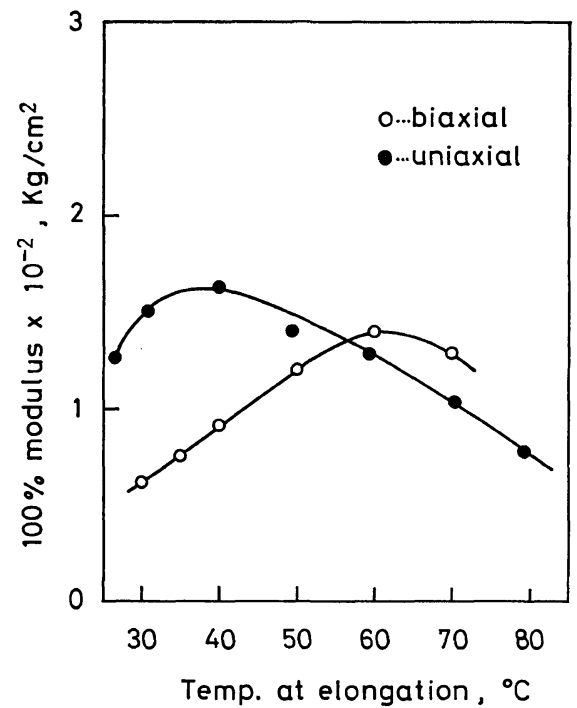

Figure 9. 100- $\%$ modulus interpolated from the curves in Figure 8 and stress-strain curves of uniaxially drawn film.

9 suggest that the stress relaxation and the plastic flow behavior $\left(\lambda_{\mathrm{r}} / \lambda_{\mathrm{m}}\right)$ are closely related to the mechanical properties of the stretched film, although they are related in a very complicated manner.

\section{ORIENTATION BEHAVIORS OF MOLECULAR CHAIN AXES}

\section{Orientation of Crystal Axes}

In a previous paper, ${ }^{1} \mathrm{X}$-ray diffraction pattern of the sample was shown and identification of the diffraction peaks was postulated for five peaks in the pattern. The first peak corresponds to (010), the second one to (200) and (110), the third to (210) and (011), and the fourth to (111) and (201).

By using the diffraction from the (010) surface, it is possible to obtain the orientation distribution of the $b$ axis. For the $a$ axis, it is impossible to obtain the orientation distribution directly from X-ray diffraction, since the separation of the intensity (200) surface from the intensity of the second peak is impossible. The combined vector $(200)+(110)$, however, is nearly parallel to the $a$ axis and both (200) and (110) vectors are in the plane containing the $a$ and $b$ axes. 
In the measurement of X-ray diffraction, the surface of the test specimen was placed in a plane perpendicular to the incident beam (horizontal) and rotated in the plane to obtain an appropriate angle $(\psi)$ between the stretching axis of the specimen and the vertical direction. Figure 10 shows the diffraction curves of a uniaxially stretched film $\left(\lambda=3\right.$, at $\left.50^{\circ} \mathrm{C}\right)$. The abscissa is the diffraction angle along $2 \theta$ scanned

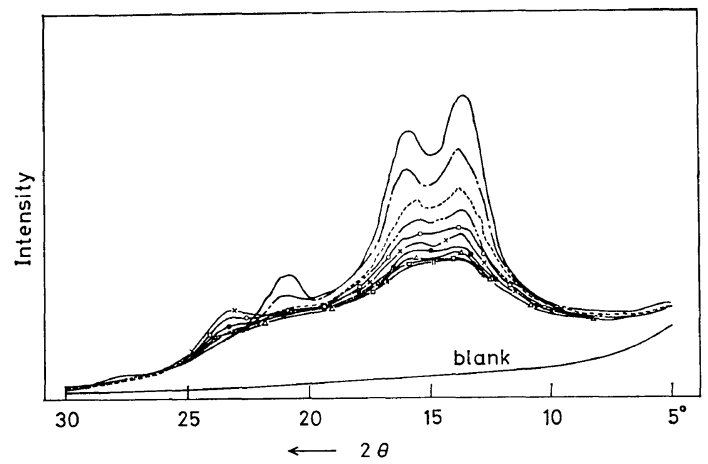

Figure 10. X-ray diffraction patterns of a specimen uniaxially drawn and kept at a constant stretch ratio $(\lambda=3.0)$ as a function of diffraction angle $2 \theta$, for fixed values of azimutual angle $\psi$ which is equal to an angle to which specimen was rotated: $\varphi=0^{\circ},(-) ; 10^{\circ},(--), 20^{\circ},(---)$; $30^{\circ}$, (-- $), 40,(-\bigcirc-) ; 50^{\circ},(-\times-) ; 60^{\circ},\left(-0^{-}\right)$; $70^{\circ},\left(-\triangle^{-}\right) ; 80^{\circ},(-\square-) ; 90^{\circ},(-\vdash)$.

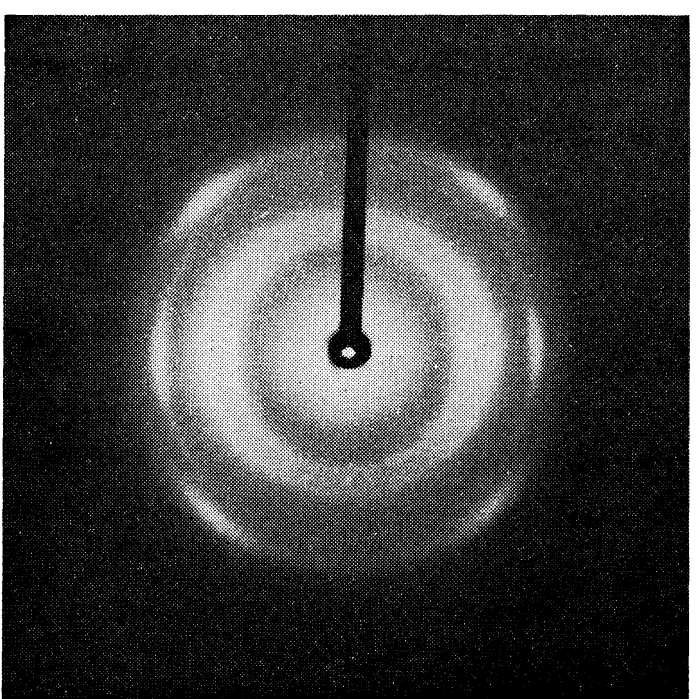

Figure 11. X-ray diffraction photographs of uniaxially drawn film $\left(\lambda=3.5\right.$, at $\left.50^{\circ} \mathrm{C}\right)$. along the equator and each curve corresponds to an individual azimuthal angle $\psi$ from zero to $\pi / 2$. Figure 11 shows $\mathrm{X}$-ray diffraction photographs of a uniaxially stretched specimen $(\lambda=5.0$, at $50^{\circ} \mathrm{C}$ ). From Figures 10 and 11 , both $a$ and $b$ axes tend to orient to directions on a plane perpendicular to the stretching direction, that is, the $c$ axis orients to the stretching direction. In order to investigate the orientation behavior quantitatively, the orientation coefficient proposed by Hermann ${ }^{6}$

$$
F_{h k l}=\frac{\left(3 \overline{\cos ^{2} \theta_{h k l}}-1\right)}{2}
$$

was calculated for some test pieces obtained by uniaxial stretching at $50^{\circ} \mathrm{C}$; this is shown as a function of strain in Figure 12.

In Figure 12 the orientation coefficients $F_{010}$ (symbolized by $F_{\mathrm{p} 1}$ in the figure) and $F_{\mathrm{p} 2}$ (which is the orientation coefficient for the combined vector $(200)+(110)$ ) have negative values and they are equal within experimental error. This fact indicates that there is no preferential orientation for the $a$ and $b$ axes. The orientation coefficient of the $c$ axis $F_{c}$ is then expressed

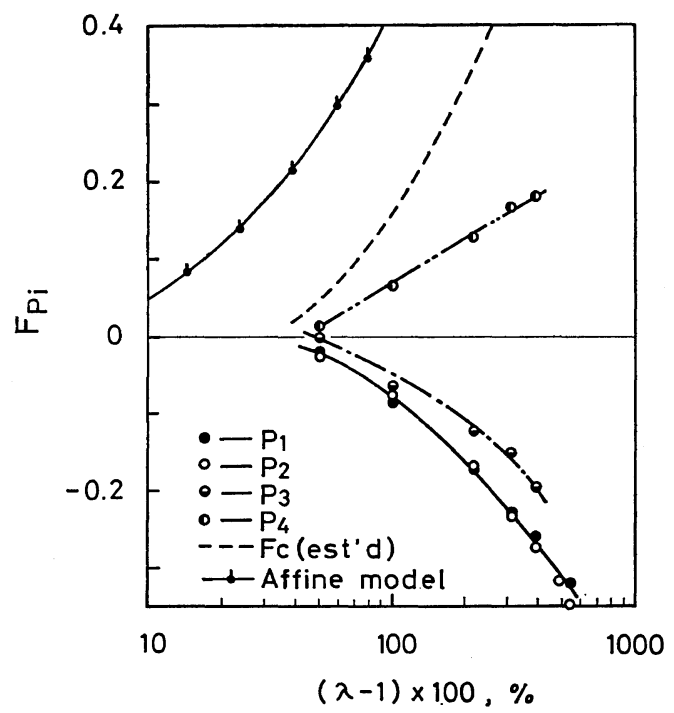

Figure 12. Orientation coefficients as a function of strain: $F_{\mathrm{P} 1}(\Theta), F_{\mathrm{P} 2}(\bigcirc), F_{\mathrm{P} 3}(\ominus), F_{\mathrm{P} 4}(\bullet)$ observed; $F_{c}$ estimated by eq $2(--)$ and $F_{c}$ calculated according to the Affine model as a function of strain (- $\left.\stackrel{1}{-}^{-}\right)$. 
by the following relation, ${ }^{5}$ by putting $F_{b}=F_{\mathrm{p} 2}$ approximately:

$$
F_{c}=-F_{a}-F_{b}=-F_{010}-F_{\mathrm{p} 2}
$$

Values of $F_{c}$ are also shown by the dotted line in Figure 12 with the values calculated by the Affine model, ${ }^{7}$ in which the $c$ axis is assumed to orient according to the deformation of the bulk specimen. It is found from the figure that values of $F_{c}$ calculated and observed are nearly superposed by a shifting along the $\log$ (strain) axis. Further, it is noted that $F_{c}$ vanishes at the strain of about $30 \%$. This critical strain $\left(\varepsilon_{\mathrm{c}}\right)$ was found to be identical with the strain at which the unrecoverable strain ratio $\lambda_{\mathrm{r}} / \lambda_{\mathrm{m}}$ becomes constant when it is observed as a function of $\lambda_{m}$ (this will be shown elsewhere). As for the dependence of the orientation function on the strain, sufficient data have been accumulated by Kawai, et al, ${ }^{8}$ to enable us to compare the results with the data of 1,2polybutadiene. The critical strain $\varepsilon_{\mathrm{c}}$ of the sample is much greater than that of polyethylene or poly(vinyl alcohol). For most of the crystalline polymers, such as low-density and highdensity polyethylenes, the dependence of $F_{a}$ and $F_{b}$ on the stretch ratio $\lambda$ are different.

This phenomena has been explained by spherulite deformation in relation to the lamella structure. Then the characteristic of the orientation of the S25 suggests the absence of a higher order crystal structure such as spherulite with lamella. This fact will be discussed elsewhere using the data of small angle X-ray and electron microscopy.

\section{Orientation of Molecular Chain in Amorphous Phase}

In order to analyse the orientation behavior of a molecular chain in an amorphous phase, the angular distribution of polarized components of fluorescence intensity were measured by an instrument of type FOM-1 manufactured by Nihon Bunko Kogyo Co. A specimen stretched and quenched in the process described in Figure 3 was clamped by a pair of chucks in the strained state, immersed in the methanol solution of fluorescent agent (commercial name of PSN) for $30 \mathrm{~min}$, and dried.

As for the analysis of the angular distribution, a precise description have been made by Nishijima, et al., ${ }^{9,10}$ on the bases of experimental and theoretical points. In this study, we did not pay much attention to a quantitative analysis of the observed angular distribution, but used it to obtain a measure of the orientation coefficient of molecular chains in amorphous phase.

In the measurement, the specimen set into the apparatus was rotated in a plane perpendicular to an incident beam in two different types of optical systems, where the directions of the polarizer and of the analyser are parallel or perpendicular to each other. The angular distributions of fluorescence intensity, designated by $I_{I /}(\gamma)$ and $I_{\perp}(\gamma)$, were measured for the respective cases, where $\gamma$ is a rotation angle set to be zero when it is parallel to the stretching direction.

In order to see how $I_{I /}(\gamma)$ and $I_{\perp}(\gamma)$ reflect the orientation distribution of the molecular chain, an imaginary case in which every orientation unit is parallel to the film plane was considered. The angular distributions are expressed as a special case of the generalized formula derived by Nishijima, et al.:

$$
\begin{aligned}
& I_{I /}(\gamma)=K \sum_{i} \cos ^{4}\left(\gamma-\omega_{i}\right) \\
& I_{\perp}(\gamma)=K \sum_{i} \cos ^{2}\left(\gamma-\omega_{i}\right) \sin ^{2}\left(\gamma-\omega_{i}\right)
\end{aligned}
$$

where $\omega_{i}$ is the orientation angle between the direction of a fluorescence molecule (which is assumed to be parallel to the molecular chain axis of the polymer) and the machine stretching direction. From these equations both $I_{/ /}(\gamma)$ and $I_{\perp}(\gamma)$ are independent of $\gamma$ for the isotropic system and $I_{I /}\left(0^{\circ}\right) / I_{I /}\left(90^{\circ}\right)$ increases when the chain molecule orients to the stretching direction.

Figure 13 shows an example of $I_{I /}(\gamma)$ and $I_{\perp}(\gamma)$ of the uniaxially stretched film $(\lambda=5.0$, stretched at $50^{\circ} \mathrm{C}$ ).

As a criterion for the orientation coefficient, the following function proposed by Y. Awaya, et $a .^{3}$ was used:

$$
\begin{gathered}
F^{\prime}=\frac{a-b}{a+2 b}, \\
a=I_{/ /}(0)+I_{\perp}(0) \quad b=I_{/ /}(90)+I_{\perp}(90) .
\end{gathered}
$$

From eq 3 and $4, F^{\prime}$ is zero for the completely random distribution and unity for complete 
Bulk Properties of Syndiotactic 1,2-Polybutadiene. II.

orientation. Figure 14 shows the values of $F^{\prime}$ as a function of the stretch ratio for respective temperatures at stretching. The figure shows

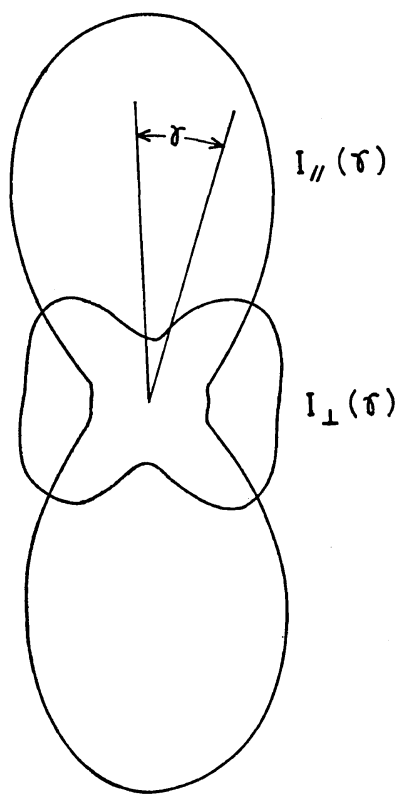

Figure 13. Angular distribution of fluorescene intensity of $I_{/ /}(\gamma)$ and $I_{\perp}(\gamma)$ of uniaxially drawn specimen (at $50^{\circ} \mathrm{C}, \dot{\gamma}=1.0 / \mathrm{min}$ ).

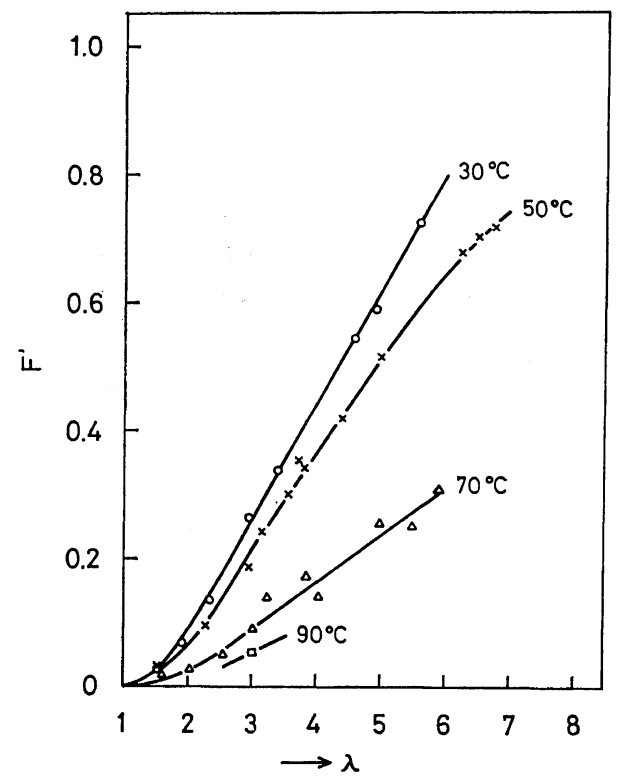

Figure 14. Orientation coefficient $F^{\prime}$ as a function $\lambda$. that $F^{\prime}$ increases with the stretch ratio and that the relation depends considerably on the temperature at stretching.

\section{CONCLUSIONS}

In Figures 8 and 9, it was shown that the mechanical property of the uniaxially or biaxially stretched specimen depends greatly on the ambient temperature at stretching. The dependence of the $100-\%$ modulus on the temperature, however, was observed to depend grestly on the type of deformation. That is, the optimum temperatures for obtaining the highest modulus were about 35 and $60^{\circ} \mathrm{C}$ for uniaxial and biaxial stretchings, respectively (Figure 9).

In order to investigate how the orientation of both crystal and amorphous chain axes contributes to the mechanical properties of the stretched specimen, the dependences of both $F^{\prime}$ and $F_{c}$ at a fixed stretch ratio $(\lambda=3$, at $\dot{\gamma}=$ $1.25 \mathrm{~min}$ ) were observed as a function of the temperature at stretching in a temperature range from 29 to $90^{\circ} \mathrm{C}$. This is the temperature range over which the melting of the crystal is observed in the DSC thermogram. ${ }^{1}$ It was found that the value of $F_{c}$ (the orientation coefficient of $c$ axis) is only slightly affected by the temperature: it decreases from 0.34 to 0.31 as the temperature changes from 30 to $90^{\circ} \mathrm{C}$. The value of $F^{\prime}$, however, showed the same temperature dependence as the 100-\% mudulus in Figure 9. The relation between $F^{\prime}$ and the 100-\% modulus is shown in Figure 15, and is seen to be linear. The extrapolation meets with the data of the original specimen (nonstretched) Pms.

We have no data available for the evaluation of the orientation under biaxial stretching. It, however, can be estimated qualitatively from other sources, such as unrecoverable strain. In Figure 15, the $100-\%$ modulus is also plotted as a function of $\lambda_{\mathrm{r}} / \lambda_{\mathrm{m}}$ for both uniaxial and biaxial data. It is noted that $\lambda_{\mathrm{r}} / \lambda_{\mathrm{m}}$ depends greatly on the type of deformation: It does, however, have a positive correlation with the $100-\%$ modulus; that is, $\lambda_{\mathrm{r}} / \lambda_{\mathrm{m}}$ has a correlation with $F^{\prime}$ if the type of stretching is fixed. These results lead to the conclusion that the orientation of the amorphous chain plays a predominant role in the formation of the mechanical properties 


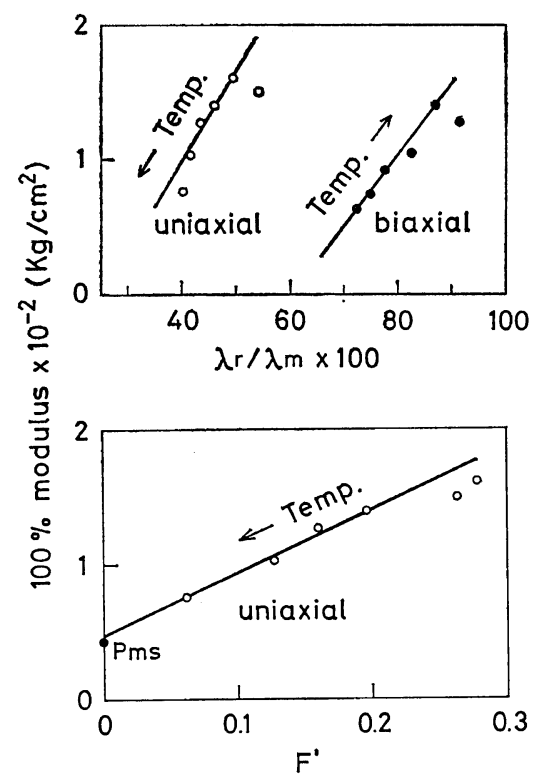

Figure 15. Dependence of $100-\%$ modulus of uniaxially and biaxially stretched film on $\lambda_{\mathrm{r}} / \lambda_{\mathrm{m}}$ and one of uniaxially stretched film on orientation coefficient $F^{\prime}$.

of stretched film in biaxial stretching as well.

Such large differences in $\lambda_{\mathrm{r}} / \lambda_{\mathrm{m}}$ and stress relaxation $f_{5} / f_{0}$ between the cases of uniaxial and biaxial stretching suggest the existence of another factor controlling the morphological change in the course of stretching.

We assumed that some kind of disintegration of the crystal occurs when a specimen was deformed biaxially. As a trial, the degree of crystallinity of a biaxially stretched specimen was measured by the heat of fusion $\Delta H_{\mathrm{f}}$ using a DSC thermogram under a constant heating rate of $16^{\circ} \mathrm{C} / \mathrm{min}$. The heat of crystallization $\Delta H_{\mathrm{c}}$ was also observed as a reference by the same test species as the one used for $\Delta H_{\mathrm{f}}$ after the melting at $160^{\circ} \mathrm{C}$, because the former reflects the effects of stretching while the latter does not. ${ }^{*}$ The values of $\Delta H_{\mathrm{f}}$ and $\Delta H_{\mathrm{c}}$ are plotted as a function of the temperatures at stretching in Figure 16. Although the data are rather scattered, it is clearly seen that the degree of crystallinity drcreases with the temperature. This

\footnotetext{
* In the Figure 16, TQ and TA indicate that the specimen was quenched or annealed after biaxial stretching in two steps.
}

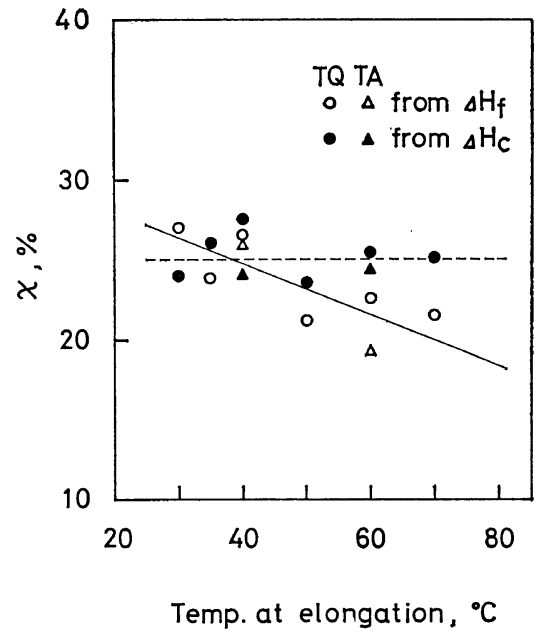

Figure 16. Degree of crystallinity obtained from the area of endothermic peak of melting process $(O, \triangle)$ and exothermic peak $(\mathbf{O}, \Delta)$ following the melting process, in DSC measurements.

result can be justified from the change of stressstrain curves of the stretched film under infinitesimal strain in Figure 8, because the modulus under infinitesimal strain is generally determined by the degree of crystallinity of the sample, as has been shown in a previous paper.

Acknowledgement. The authors acknowledge the helpful coments made by Drs. K. Yagii, M. Niinomi, T. Homma, and K. Ninomiya. The authors also wish to express their gratitude to Dr. T. Hashimoto for discussions on the orientation of molecular chain axes.

\section{REFERENCES}

1. Y. Obata, C. Tosaki, M. Ikeyama, and C. Homma, submitted to Polymer. $J$.

2. Y. Takeuchi, M. Ichikawa, and K. Mori, Paper presented at 5th Polymer Symposium, Osaka Japan.

3. H. Awaya and T. Isobe, Kobunshi Kagaku (Chem. High Polymers), 29, 196 (1972).

4. Y. Obata, S. Kawabata, and H. Kawai, J. Polym. Sci., 38, 2997 (1970).

5. R. S. Stein, ibid., 31, 327 (1958).

6. P. H. Hermans and P. Platzeck, Kolloid-Z. Z. Polym., 88, 68 (1938).

7. R. S. Moore, J. Polym. Sci., 5, 711 (1967).

8. H. Kawai, "Proc. 5th Int. Cong. Rheo.", 
Bulk Properties of Syndiotactic 1,2-Polybutadiene. II.

Vol. 1, 1969, p 97.

9. Y. Nishijima, Kobunshi (High Polymers, Japan), 15, 868 (1966).
10. Y. Onogi and Y. Nishijima, Rep. Progr Polym. Phys. Japan, 14, 537 (1971).

11. Y. Obata, unpublished data. 